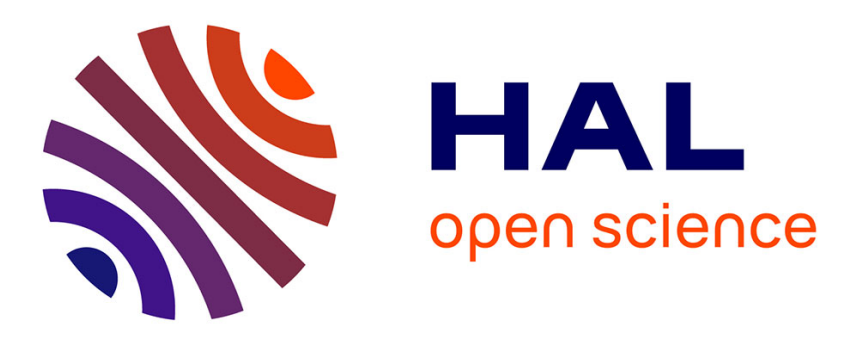

\title{
Clear-water scouring process in a flow in supercritical regime
}

Emmanuel Mignot, T. Moyne, Delphine Doppler, Nicolas Rivière

\section{To cite this version:}

Emmanuel Mignot, T. Moyne, Delphine Doppler, Nicolas Rivière. Clear-water scouring process in a flow in supercritical regime. Journal of Hydraulic Engineering, 2016, 142, pp.04015063. 10.1061/(ASCE)HY.1943-7900.0001100 . hal-01516087

\section{HAL Id: hal-01516087 \\ https://hal.science/hal-01516087}

Submitted on 21 Nov 2018

HAL is a multi-disciplinary open access archive for the deposit and dissemination of scientific research documents, whether they are published or not. The documents may come from teaching and research institutions in France or abroad, or from public or private research centers.
L'archive ouverte pluridisciplinaire HAL, est destinée au dépôt et à la diffusion de documents scientifiques de niveau recherche, publiés ou non, émanant des établissements d'enseignement et de recherche français ou étrangers, des laboratoires publics ou privés. 


\title{
1 Clear-water scouring process in a flow in supercritical regime
}

Mignot E. ${ }^{*}$, Moyne T. ${ }^{2}$, Doppler D. ${ }^{3}$ and Riviere N. ${ }^{4}$

${ }^{1}$ Assistant professor, LMFA, CNRS-Université de Lyon, INSA de Lyon, Université Claude Bernard Lyon

1, Ecole Centrale de Lyon, emmanuel.mignot@insa-lyon.fr

${ }^{2}$ Master student, LMFA, CNRS-Université de Lyon, INSA de Lyon, Université Claude Bernard Lyon 1, Ecole Centrale de Lyon, thomas.moyne@gmail.com

${ }^{3}$ Assistant professor, LMFA, CNRS-Université de Lyon, INSA de Lyon, Université Claude Bernard Lyon 1, Ecole Centrale de Lyon, delphine.doppler@univ-lyon1.fr

${ }^{4}$ Professor, LMFA, CNRS-Université de Lyon, INSA de Lyon, Université Claude Bernard Lyon 1, Ecole Centrale de Lyon, nicolas.riviere@insa-lyon.fr

\begin{abstract}
The aim of this research is to measure the clear-water scouring produced by supercritical flow around a rectangular shaped obstacle. The initial uniform flow condition without the obstacle is such that the Shields parameter remains slightly lower than critical, so that the sediment constituting the mobile bed is not transported. After the obstacle is suddenly inserted, the sediment begins to move. At specific times after this the 2D water surface and the 2D bed topography fields around the obstacle are then measured. The initial flow pattern exhibits a typical bow-wave like hydraulic jump, detached upstream from the obstacle, occurring over the rough plane bed. Then, as for the more widely studied subcritical regime configuration, the scour initiates on the sides of the obstacle and migrates towards its upstream face, where the scour depth continues to increase with time. This causes the hydraulic jump to migrate downstream, approaching the obstacle with negligible effect on the bed topography. Downstream from the obstacle, the sediment deposits and forms a specific deposition zone whose maximum elevation also increases with time before reaching a plateau. The flow pattern downstream of the obstacle is strongly influenced by this deposition and exhibits two consecutive bow-waves aligned in the streamwise direction with the hydraulic jump located upstream. The experiment runs for two hours and even though all evolution rates decrease with time, this duration is not sufficient to obtain a stable situation of the flow pattern and bed topography. The paper ends with a similarity analysis of the typical field conditions for which this clear-water scouring process could occur.
\end{abstract}




\section{Introduction}

Open-channel flows around obstacles and subsequent scour formation at the toe of the obstacle has been widely studied, mostly due to its importance for bridge pier or abutment stability. The experiments available in the literature and cited in the papers below were mostly focused on steady subcritical flows over fixed or mobile beds and for mobile bed experiments, under livebed or clear-water configurations. Much less research has been devoted to such flows in supercritical regimes, but the published results are then summarized in the following section.

\section{Subcritical flow over a fixed or mobile bed}

The most studied configuration is that of subcritical flow over a (usually smooth) fixed-bed. As the incoming flow approaches the obstacle, the adverse pressure gradient between the flow upstream and the obstacle leads to a boundary layer separation in the near-bottom region and the creation of a horseshoe vortex at the toe of the obstacle on its upstream side. Graf and Yulistiyanto (1998), Ahmed and Rajaratnam (1998), and Roulund et al. (2005) described the flow patterns upstream of the obstacle and within the horseshoe vortex. The experiments of Roulund et al. (2005) and Sadeque et al. (2008) showed that the bed shear stress is negative far upstream from the obstacle, goes to zero, and finally becomes positive as the obstacle is approached. The location at which the bed shear stress reaches zero corresponds to the separation point of the boundary layer and thus the upstream limit of the horseshoe vortex. Dargahi (1989), Sahin et al. (2007) and Escauriaza and Sotiropoulos (2011) showed that, depending on the inflow Reynolds number, the horseshoe vortex can consist of a single or multiple vortices.

In experiments with a mobile bed without sediment recirculation, usually referred to as 'clearwater', the flow condition is selected so that the Shields parameter remains lower than critical and thus no sediment transport takes place in the absence of the obstacle (and, therefore, in the far field). Authors such as Muzzammil and Gangadhariah (2003), Unger and Hager (2007), Dey and Raikar (2007), Kirkil et al. (2008), Gober et al. (2010), Diab et al. (2010) or Link et al. (2012) describe the scouring process taking place around the obstacle. The horseshoe vortex appears in front of the obstacle at the very beginning of the experiment, and the scour initiates at an angle of about $75^{\circ}$ to the channel axis due to the accelerating flow along the sides of the obstacle. Then the scour extends towards the upstream face of the obstacle that is towards the horseshoe vortex which is replaced by a downflow within the developing scour with complex vortex dynamics (see Kirkil et al. 2008 or Link et al. 2012). The depth and upstream extension of the scour then increase and a larger scale vertical recirculating flow occurs within the scour hole, composed of one or more coherent vortices. According to Hager and Unger, (2010), "it is currently accepted that pier scour advances logarithmically with time and that an equilibrium scour depth is hardly attained, except for weak approach flow conditions or a non-uniform 
sediment". It is important to note that Dey and Raikar (2007) and Diab et al. (2010) showed that the scour depth is increased and that the scour progresses more slowly, and with steeper slopes if the cylindrical obstacle is replaced by a square of sides equal to the circular pier diameter. Downstream from the pier, the flow initially separates and a wake with recirculating flow forms a zone of deposition immediately downstream from the scour zone (Kirkil et al. 2008). As time progresses, the separation point moves downstream (see Oliveto and Hager, 2014) and this recirculation zone becomes negligible. The maximum deposition height in the downstream zone remains limited.

Live-bed scouring experiments are performed with a Shields parameter exceeding the critical value, so that sediment transport takes place over the bed even without (and far from) the obstacle, and an additional flow loop to recirculate the suspended sediment. The methodology followed by Jain and Fischer (1980), Chiew (1984), Melville and Chiew (1999) or Sheppard and Miller (2006) is to gradually increase the inflow velocity and measure the corresponding equilibrium scour depth. As this velocity increases, "a peak in the equilibrium scour depth [is observed] at the transition from clear-water to live-bed conditions, a decrease in equilibrium scour depth [is observed] just beyond this peak, and a second peak in the live-bed scour range (believed to occur where the bed 'planes out')" (Sheppard and Miller 2006). According to Jain and Fischer (1980), this process is the result of a competition between: i) the increasing sediment transport from upstream region where erosion takes place as the critical Shields parameter is exceeded and ii) the increasing strength of the scour mechanism to dislodge particles from the bottom of the scour hole. Visualization or velocity data acquisition in such suspended sediment flow is delicate and most authors restrict their data to the scour depth evolution and magnitude in the 'equilibrium' state.

Additionally, effects of unsteadiness have been investigated in laboratory, clear water conditions by authors such as Lai et al. (2009) or Hager and Unger (2010) or in field, flood conditions by Lu et al. (2008).

\section{Supercritical flows over a fixed or mobile bed}

All previously cited papers (and the extensive accompanying literature) deal with flows in the subcritical regime, obviously related to the fact that most bridge piers are constructed in rivers where the flow regime remains subcritical even during floods. However, in mountainous areas the flow can become nearly critical or even supercritical. For instance, in areas like the French island, La Reunion in the Indian Ocean or Chile, the river flow can be supercritical even during normal hydrological conditions, and reach high Froude numbers after heavy rains, resulting in a significant increase in sediment transport.

Much less research has been reported on the interaction between a supercritical open channel inflow and a bridge pier. Jiang and Smith (2000) reported that a stationary shock wave forms in 
front of the obstacle, shaped as a bow wave near the centerline and a V-wave further away. Near the centerline (the symmetry axis), the shock is perpendicular to the flow axis, with a subcritical flow on the downstream side of the jump deflected away from the centerline, passing on either side of the obstacle. Further from the centerline, the supercritical inflow experiences an oblique jump at the trailing edge of the $\mathrm{V}$-wave and the flow downstream from the jump remains supercritical with a deflected flow direction, as predicted by Ippen (1951). This flow pattern is highly similar to that observed in aerodynamic studies of detached shock waves upstream of bluff bodies (see, for example, Shapiro, 1953). More recently, Mignot and Riviere (2010) and Mignot et al. (2011) have investigated the interaction between a supercritical open channel inflow over a smooth bed and a rectangular shaped obstacle, focusing on both flow structures which occur in front of the obstacle: the detached hydraulic jump and the horseshoe vortex. This research shows that if the inflow is turbulent, then the detachment length of the hydraulic jump exceeds the detachment length of the horseshoe vortex. Along the centerline, the flow first reaches the detached, bow-like, hydraulic jump, undergoes transition to the subcritical regime and then reaches the horseshoe vortex which acts as a positive step, leading to a sudden increase of the water depth (see Fig. 1). Finally the flow is deflected around the obstacle. The authors also adapted the analytical model developed by Mockel (1949) for the shape and location of the detached shock wave in a supersonic flow around a bluff body to the present supercritical, open-channel, flow in order to predict the evolution of the dimensionless detachment length of the hydraulic jump as the Froude number of the inflow increases. Finally, in a further study, Riviere et al. (2012) observed a complete modification of the previously reported flow pattern as the turbulent intensity of the incident flow is increased by an additional flow disturbance (a von Karmann vortex street from a narrow obstacle introduced upstream along the centerline and by removing the upstream stilling device). The detachment length of the hydraulic jump is not affected but the length of the horseshoe vortex increases so that both detached structures occur at the same distance from the obstacle.

A few studies dealt with scour resulting from supercritical flow around an obstacle such as studies from Jain and Fischer (1980) or Chiew (1984). At the highest values of the inflow velocity, the upstream Froude number exceeds 1 (with values up to 1.5), but the authors did not report specific modifications in the flow pattern or the scour depth tendency. In this study, the determining factor seems to be the Froude number at which the critical Shields parameter is reached (lower than 1) and for which the configuration passes from clear-water to live-bed, rather than Froude equal to 1. It is important to note that for the supercritical flow configurations tested by the authors, the Shields parameter exceeds the critical value so that the flow around the pier is considered as 'live-bed' and antidune fields were observed. The authors 
provide an empirical relation between the maximum scour depth and the difference between the inflow Froude number and the critical Froude number.

\section{Present work}

The present research differs from the studies cited above as it is performed in clear water conditions, as it is dedicated only to the supercritical regime and as its objectives differ. The first objective of the present work is to verify whether the flow pattern observed by Mignot and Riviere (2010) over a fixed smooth bed also occurs with a rough bed and how this flow pattern evolves with time as the scour evolves. The second objective is to measure and analyze the dynamics of the scouring and deposition processes around the obstacle in the presence of a detached hydraulic jump and compare them with the subcritical configurations reported in the literature. In the present clear water experiments, the absence of bed forms such as the ones reported in the cited live bed experiments allows to focus on this hydraulic jump/scour hole interaction. The experimental set-up is first presented along with all measurement devices. Then the time-evolution of both bottom topography and flow pattern are presented and discussed. Finally, the similarity of the present flow with natural flows is discussed.

\section{Experimental approach}

\section{Experimental set-up}

The experiments have been conducted in an open-channel flume located in the Laboratory of Fluids Mechanics and Acoustics (LMFA) at the University of Lyon (INSA Lyon, France). The flume sketched in Fig. 2a is $1.4 \mathrm{~m}$ long, straight, with a rectangular cross-section of width $b=0.49 \mathrm{~m}$ and a constant streamwise slope of $i=6.7 \%$. The discharge $Q=2.87 \mathrm{~L} / \mathrm{s}$ is measured in the pumping loop using an electromagnetic flowmeter (Endress-Hauser) with an uncertainty $\pm 0.05 \mathrm{~L} / \mathrm{s}$. The upstream boundary condition consists of a grid buffer and a honeycomb with a small mesh in order to stabilize the inflow. The downstream boundary condition is a critical depth. The uniform water depth in the channel then equals $h_{n}=9.35 \mathrm{~mm}$ (precision of $0.05 \mathrm{~mm}$ ). The inflow velocity equals $U=Q / b h_{n}=0.63 \mathrm{~m} / \mathrm{s}$, the Froude number equals $F r=2.07$ and the Reynolds number $R e=2.3 \times 10^{4}$.

The selected sediment is composed of blasting shot - steel - balls, (approximately spherical) of diameter $d=1.5 \mathrm{~mm}$ and dispersion $\sigma=\left(\mathrm{d}_{84} / \mathrm{d}_{16}\right)^{1 / 2}=1.06$, and density $\rho_{\mathrm{s}}=7400 \mathrm{~kg} / \mathrm{m}^{3}$, shown in Fig. 2 d, leading to a vertical aspect ratio of $h_{n} / d=6.2$. It is important to note that within the sediment there are some clusters formed by two or three balls that have fused together. Along the first $50 \mathrm{~cm}$ of the channel, the bed level is increased by a step of $19 \mathrm{~mm}$ and is covered by one layer of glued sediment (Fig. 2). The length over water depth ratio for this upstream channel reach is equal to 53, ensuring the establishment of the supercritical uniform flow at inlet. The 
downstream reach is $40 \mathrm{~cm}$ long, and is similar to the upstream reach. The mobile bed section thus occupies the central reach, $50 \mathrm{~cm}$ long (over the $b=49 \mathrm{~cm}$ channel width). The rectangular chamber is filled with sediments over a layer of about $20.5 \mathrm{~mm}$ (Fig. 2b), initially flattened by a moving plate. The mean sediment elevation is used as vertical elevation reference $z=0$.

The bed shear stress estimated in the uniform flow conditions (without the obstacle) equals: $\tau=\rho_{w} g h_{n} i=6.15 \mathrm{~N} / \mathrm{m}^{2}$ and the Shields parameter - corrected by factor $\eta$ for high slopes following the recommendations from Cheng and Chen (2013) - equals $\theta=\frac{\tau}{\left(\rho_{s}-\rho_{w}\right) g d} / \eta=$ 0.048 with a dimensionless particle diameter of $d_{*}=d\left[\frac{\left(g \rho_{s}-g \rho_{w}\right)}{\rho_{w} v^{2}}\right]^{1 / 3}=60$. The corresponding critical Shields parameter on Shields diagram equals $\theta_{\mathrm{cr}} \sim 0.045$ with $\rho_{\mathrm{w}}$ and $v$ the water density and kinematic viscosity respectively. The uniform flow is thus very close to the incipient motion; this was verified by two observations:

i) after the few over-exposed sediment grains have been removed the bed remains stable for long times

ii) a slight increase in the discharge or the slope results in significant sediment motion.

The obstacle is an impervious rectangular-shaped empty box with splayed corners, open along its bottom face of length (along the flow axis) $\mathrm{L}=65 \mathrm{~mm}$ and width (along the transversal axis) $R=98 \mathrm{~mm}$. The borders are sharp so that the obstacle can be inserted rapidly into the water and through the sediment bed, all the way down to the rigid bed of the flume, with very little disturbance of the sediment bed. It was verified (by eye) that inserting the obstacle did not cause any sediment motion and this is corroborated by the fact that negligible change of topography is measured in the 10 first seconds following the obstacle insertion. A specific device attached to the flume ensured that the obstacle location was the same in each experiment. The $x=y=0$ origin is located at the center of the obstacle with $x$ aligned with the principal flow direction and $y$ with the transverse direction.

\section{Measurement devices}

The bed topography and water level are defined along the $z$ axis (referred to as "vertical axis") which is perpendicular to the bed (differing from the terrestrial vertical due to the bottom slope $i=6.7 \%)$. The same laser grid technique is employed for measuring both the bed topography and the free surface elevation, using slightly different procedures. For measuring the bed topography, the flow is stopped by switching off the pump and blocking the inflow, the water evacuates through a reverse flow in the pump towards the downstream tank and the topography is measured once the bed is dry. The free surface elevation is measured at constant discharge, by mixing a small amount of white dye in the water to render the water opaque. 
The measurement method is detailed in the Appendix. Bed topography and free surface elevation were measured at two locations - one upstream of the obstacle and one downstream; the measurement zones overlapped slightly to provide some verification of the reproducibility of the results. Taking advantage of the symmetry of the flow, only the left side of the domain was measured, with a spatial resolution of about $15 \mathrm{~mm}$.

\section{Procedure}

The procedure used is as follows:

1- the plane sediment bed reference level $(z=0)$ is established, with the bed flattened as described above.

2- the pump is switched on and steady uniform flow is established, without the obstacle. Prominent, exposed grains are rapidly washed away by the flow.

3- the obstacle is inserted rapidly, time is set to $t=0$ and measurements start. Two different procedures were used for the free surface and the topography measurements:

3a- for the free-surface measurement, photos of the laser grid intersection on the opaque water surface are taken at fixed times and the free-surface is later reconstructed for all times. This procedure involving steps 1, 2 and $3 a$ is performed twice, once for each zone.

$3 \mathrm{~b}$ - for the topography measurement, the flow is suddenly stopped after the desired running time by blocking the flow through the upstream honeycomb; once the bed is sufficiently dry, a photo of the laser grid on the sediment bed is taken. The whole procedure involving steps 1,2 and $3 \mathrm{~b}$ is then repeated for a different running time.

Reproducibility of the scouring process was verified by repeating the procedure several times and comparing the results. The impact of stopping the flow in step $3 \mathrm{~b}$ was verified by comparing the bed topography i) measured after 40 minutes of continuous flow and ii) measured after 4 consecutive series of 10 minutes of flow (with thus four consecutive stops). The differences in bed topography after 40 minutes were limited and negligible relative to the total evolution as highlighted in Fig.3. This method appears to underestimate slightly the water depth because the water is not fully opaque (unlike the sediment); when the laser grid is projected onto the freesurface, the light penetrates the water slightly before being reflected, so that the image spots are actually located somewhere within the upper layer of the water (see, for example, Lipeme Kouyi et al., 2003).

\section{Results}

Flow pattern at initial time.

As the obstacle is introduced at $t=0 \mathrm{~s}$, the bed is flat and the flow pattern (not shown here) is very similar to the one at $t=0.5$ min in Fig.3: a detached hydraulic jump of hyperbolic shape forms 
upstream of the obstacle as described by Mignot and Riviere (2010). The detachment length $\lambda$ of the toe of the jump along the centerline $(y=0)$ equals about $80 \mathrm{~mm}$, that is $\lambda / R \sim 0.8$ which is of same order of magnitude as the detachment length measured for a Froude number of 2 by Mignot and Riviere (2010) (see their figure 12a). As shown by Riviere et al. (2012, figure 4) the water depth and water level increase with downstream distance, reaching a maximum elevation of about $z_{w}=h=30 \mathrm{~mm}$ at the upstream face of the obstacle. The free surface on either side of the centerline evolves in a similar fashion but with a more limited maximum elevation $\left(z_{w}=15 \mathrm{~mm}\right.$ for $y=-180 \mathrm{~mm}$ ). The hydraulic jump reflects at the left bank (located at $y=-245 \mathrm{~mm}$ ) and a crosswave is observed for $x>100 \mathrm{~mm}$. Behind the obstacle, the water depth and water level are very low, so that the bed is almost dry just downstream from the obstacle along the centerline.

\section{Evolution of the bed topography}

As in the subcritical regime, the scour starts along each side of the obstacle with a maximum scour depth of $4 \mathrm{~mm}$ at $t=30$ s extending over the whole side of the obstacle (along $x$ axis) and a width of $30 \mathrm{~mm}$. At the location of the detached hydraulic jump, no erosion is observed, as revealed by line $\mathrm{L} 1$ for $x \sim-50 \mathrm{~mm}$ at $t=30 \mathrm{~s}$ in Fig. 4 .

As time progresses, the scour extension increases towards upstream, downstream and in the transverse directions, as revealed by L2 and L3 lines in Fig.4, but the scour always remains more extended towards downstream, than upstream. After $t=1.5$ minutes, the scour reaches the upstream corner of the obstacle and starts to extend along its front face; the scour reaches the centerline $y=0$ at $t \sim 3 \mathrm{~min}$. Nevertheless, the maximum scour depth remains located on the side of the obstacle ( $x=0$ and $y=-R / 2$ ) and reaches about $-10 \mathrm{~mm}$ after 3 minutes and $-15 \mathrm{~mm}$ after 30 minutes (see line L3). After $\mathrm{t}=30 \mathrm{~min}$, the maximum scour depth does not increase much further but the area of the deep scour region keeps on increasing: the $z_{b}<-10 \mathrm{~mm}$ region reaches the centerline at $t \sim 60 \mathrm{~min}$ and a thin layer of $z_{b}<-14 \mathrm{~mm}$ is observed along a semicircle around the upstream half of the obstacle at $\mathrm{t}=120 \mathrm{~min}$ on Fig.5.

In the meantime, a deposition region forms and grows with time in the wake of the obstacle at $x \sim 100$ to $150 \mathrm{~mm}$ and $y \sim 20-30 \mathrm{~mm}$, as revealed by line L5. This deposition region grows upstream and reaches the back face of the obstacle at $t \sim 3 \mathrm{~min}$ but the increasing extension of the scour in this region limits the extension of the deposition zone for $t>10 \mathrm{~min}$. This deposition takes place on both sides of the centerline but Figs. 3 and 4 reveal that along the centerline itself (along L1 at $y \sim 0$ ), the bed elevation remains almost unchanged. The deposition pattern strongly differs from the deposition patterns observed for subcritical flow configurations for which a dune aligned with the centerline is observed downstream from the obstacle (see Kirkil et al., 2008 or Oliveto and Hager 2014). As a consequence, after $t \sim 120 \mathrm{~min}$, a complex pattern of flow deposition and scour takes place with both regions of about the same extension but the scour depth is about twice the deposition elevation (see Fig.6). 
Fig.6 shows the time evolution of the maximum measured erosion depth and deposition height around the obstacle. Both maxima appear to increase following a somewhat logarithmic curve during the first 10 minutes with a higher slope for the scour depth than for the deposition elevation. Afterwards, the maximum erosion depth and deposition elevation reach a plateau.

\section{Evolution of the flow pattern}

In this section, the time evolution of the flow pattern is described separating the domain into four regions.

i) Upstream from the obstacle, the detached hydraulic jump approaches the obstacle. As revealed by line L1 (Fig.4) for negative $x$ values, the $z_{w}=20 \mathrm{~mm}$ elevation moves from $x=-80 \mathrm{~mm}$ at $t=30 \mathrm{~s}$ to $x=-50 \mathrm{~mm}$ at $t=3 \mathrm{~min}$. Upstream from the jump, the water depth then becomes equal to the upstream uniform depth, as at $t=5 \mathrm{~min}$ at $x \sim-100 \mathrm{~mm}$. Since, as shown above, the jump does not affect the bed elevation as it moves downstream, the extension of the region impacted by the hydraulic jump decreases and is replaced by a flat bed with uniform flow. It can also be seen that, as time progresses, the scour depth increases in front of the obstacle and thus, whilst the water level does not change, the water depth strongly increases in this region and exceeds $40 \mathrm{~mm}$ at $t \sim 60 \mathrm{~min}$.

ii) along the sides of the obstacle, the crest of the hydraulic jump also moves downstream and towards the obstacle (along the transverse axis). At $t=30 \mathrm{~s}$, the crest is located at $x \sim 55 \mathrm{~mm}$ along $y=-180 \mathrm{~mm}$ and at $x \sim-25 \mathrm{~mm}$ along $y=-100 \mathrm{~mm}$ (along L2) and at $t=3 \mathrm{~min}$, it moves to $x \sim 100 \mathrm{~mm}$ $(y=-180 \mathrm{~mm})$ and $x \sim 0 \mathrm{~mm}(y=-100 \mathrm{~mm})$ respectively. Meanwhile, the crest elevation decreases (see L2). This behavior is confirmed by the displacement of the maximum elevation along line L3 towards the obstacle. As the jump moves downstream and towards the obstacle, the location previously occupied by the hydraulic jump remains at its initial elevation $\left(z_{b} \sim 0\right.$, as can be seen along L2 and L3) and the flow there becomes uniform (the water depth equals the normal depth as prior to the introduction of the obstacle). Again, the extent of the region affected by the obstacle diminishes towards the obstacle.

iii) still along the sides of the obstacle, in the region just downstream from the hydraulic jump, the bed remains quite flat at its initial elevation for $t<10 \mathrm{~min}$ and the water depth equals the water level except along the lateral face of the obstacle where the scour develops and where the water level decreases (see L3) but not enough to ensure a constant water depth which in turn increases. Later on $(t>10 \mathrm{~min})$, the flow pattern becomes more complex and is detailed in the following paragraph.

iv) downstream from the obstacle, for $t \sim 30 \mathrm{~s}$, the deposition elevation is negligible and the water depth and water level decrease significantly. Later, for $t<5 \mathrm{~min}$, the deposition elevation increases and the water level and water depth slightly increase. Then, as the deposition elevation exceeds $6 \mathrm{~mm}$ locally, the water level remains high but the water depth strongly 
decreases; the region far downstream from the obstacle becomes almost dry. For $t \geq 30 \mathrm{~min}$, the deposition zone covers a considerable area, with significantly increased elevation, so that this deposition zone acts as a 'second obstacle' located downstream of the main one. As a consequence, a second bow wave (similar in shape to a hydraulic jump) forms parallel to the first one, located about $100 \mathrm{~mm}$ downstream, but with a maximum crest elevation displaced to the side at $y \sim-60 \mathrm{~mm}$. It can also be observed, Fig.3 (for $t=60 \mathrm{~min}$ ), Fig.4 (along L2), Fig.5 $(t=120 \mathrm{~min})$ and the photo in Fig.7 ( $t \sim 90 \mathrm{~min})$ that a third elevated depth zone forms at about $100 \mathrm{~mm}$ downstream from this second one. The present measurements do not allow us to determine whether these two bow-waves (located at $x=100 \mathrm{~mm}$ and $x=200 \mathrm{~mm}$ along L2 in Fig.4) are hydraulic jumps (with a local Froude number passing from higher to lower than 1 ) or just gravity waves.

\section{Evaluation and measurement of the sediment volume leaving the flume}

The bed volume (eroded within the $50 \mathrm{~cm}$ long and $49 \mathrm{~cm}$ wide mobile bed region) is evaluated using two approaches i) directly from a 2D integral of the bed topography at different times (see Fig.3) and ii) by measuring the weight of sediment caught in a sediment trap located at the outlet of the flume. This second technique requires relating the weight of the dried sediment with the equivalent bed volume. This relation is obtained by setting up a $20.5 \mathrm{~mm}$ high bed as for all experiments and running the flow without obstacle to let the over-exposed sediment elements leave the flume (see the experimental set-up section) then collecting all the sediments from this mobile bed region, drying and weighing them.

Fig.8 reveals that the results from the two methods agree and that at initial time, the erosion process is very intense and this intensity rapidly decreases with time. The erosion rate appears to follow a power law reasonably closely for the 5 first minutes, followed by a more gentle constant rate until the end of the experiment $(t=120 \mathrm{~min})$.

\section{Discussions}

\section{Analysis of the hydraulic jump displacement}

The aim of this section is to discuss the reason why the hydraulic jump moves downstream towards the obstacle.

The jump displacement can be analyzed based on the mass conservation balance proposed by Moeckel (1949) and adapted to open-channel flow by Mignot and Riviere (2010). In Fig.9a, the blue line represents the jump, whose detachment length along the symmetry axis is noted $\lambda$; the section of length $L_{o}$ corresponds to the minimum flow section, where the Froude number thus equals 1 ( $L_{o}$ thus corresponds to the critical section). Flow in the region enclosed by i) the jump, ii) the vertical symmetry plane, iii) the obstacle and iv) the section of length $L_{o}$ is subcritical 
$(F r<1)$ whilst elsewhere the flow is supercritical $(F r>1)$. The flow rate entering the subcritical region is noted $Q_{i}$ with $Q_{i}=L_{i} h_{i} U_{i}$ with $h_{i}$ and $U_{i}$ the normal water depth and corresponding velocity of the uniform flow upstream. The discharge leaving this region through $L_{o}$ is noted $Q_{o}$ with $Q_{o}=L_{o} h_{o} U_{o}=L_{o} h_{o}^{3 / 2} g^{1 / 2}$ (as $F r_{o}=1$ ) and $h_{o}$ the mean water depth along $L_{o}$. Thus the continuity equation can be written:

As time progresses, we consider that $h_{i}$ and $U_{i}$ remain unchanged as the bed is not affected by the hydraulic jump (as discussed above, see for instance line L1 in Fig. 4). Consequently, from Eq (1) $h_{o}^{3 / 2} L_{o} / L_{i}$ must remain constant. Fig. 3 reveals that $h_{o}$ increases with time as the scour develops near the upstream side corner of the obstacle; consequently the ratio $L_{o} / L_{i}$ has to decrease. The sketch in Fig.9b reveals that, from geometrical considerations, if $\lambda$ increases from $\lambda_{1}$ to $\lambda_{2}$ the increase of $L_{o}$ (from $L_{o 1}$ to $L_{o 2}$ ) greatly exceeds that of $L_{i}$ (from $L_{i 1}$ to $L_{i 2}$ ) so that the ratio $L_{o} / L_{i}$ increases; conversely if $\lambda$ decreases from $\lambda_{2}$ to $\lambda_{1}, L_{o} / L_{i}$ must decrease as required by continuity. Based on this analysis, the scour process developing in the vicinity of the side corners of the obstacle is responsible for the hydraulic jump approaching the obstacle. vortex also influences the position of the hydraulic jump: the horseshoe vortex modifies the 'effective' channel bottom, which incidentally makes it impossible to close Eq (1). In the present configuration, as time increases, the scour in front of the obstacle increases and so the horseshoe vortex may become confined within the scour, as previously described by Kirkil et al. (2008), and the corresponding discussion by Gobert el al. (2010), or more recently by Link et al. (2012). The main flow should then take place «above» the horseshoe vortex whose impact on the hydraulic jump becomes negligible. This also affects the jump detachment length.

\section{Comparison of scour geometry with subcritical cases}

The subcritical scour configuration closest to the present configuration seems to be the one from Diab et al. (2010) who considered a square pier (in their figures 3 and $4 \mathrm{~b}$ ), in the clear-water regime with gravel as sediment, instead of the finer sediment usually used in other studies. One important difference is that in the present case the obstacle is rectangular and not square. Figure $4 \mathrm{~b}$ from Diab et al. (2010) shows that, in subcritical conditions, the maximum scour depth initially occurs at an angle of about $45^{\circ}$ to the mean flow axis and then moves towards the symmetry plane in the upstream region after about $\mathrm{T}=5340$, with $\mathrm{T}$ the dimensionless scour time, as defined by Oliveto and Hager (2002). Oppositely, in the present case, Fig.10 reveals that the maximum scour depth starts on the side of the obstacle (angle equal to $90^{\circ}$ ) and remains at this location for long times, until at least $t=120$ minutes ( $T=36500$, with $T \sim 5.07 t$ in the present study, with $t$ expressed in seconds) where the maximum scour depth becomes about equal along the upstream and side faces of the obstacle (angles of $0^{\circ}, 45^{\circ}$ and $90^{\circ}$ ). Another major difference 
is that, in this study, at intermediate times ( $t=5$ to $10 \mathrm{~min}$, that is $T=1520$ to 3040 ), the scour along the upstream face (angle of $0^{\circ}$ ) is negligible whilst it is already deep on the side of the obstacle $\left(z_{\mathrm{b}}=-12 \mathrm{~mm}\right.$ at an angle of $\left.90^{\circ}\right)$; in comparison, Diab et al. (2010) found that at such time ( $T=1920$ to 3240), the scour depth along the upstream face is about equal to that along the side face. A third major difference lies in the downstream region where, in subcritical conditions, Diab et al. (2010) found that the maximum scour depth is about half that of the upstream region (for $T \sim 11760-72540$ ), whilst in the present supercritical configuration, the scour remains negligible at least during the first 120 minutes $(T=36500)$, as shown in Fig. 4. The final major difference between scouring in subcritical and supercritical inflows concerns the influence of the channel to obstacle width ratio $b / R$. In this study, the obstacle and the jump are separated from the side walls by a region where the flow is both fully-developed and in the supercritical regime. The characteristics of this flow depend only on the upstream conditions and bottom slope and are thus completely independent of the position of the side walls. As long as the hydraulic jump intersects with the side walls downstream from the rear of the obstacle (as in the present case, see Fig. 3), the side walls will not influence the scouring process in the near-obstacle region, and there is no need to consider the blockage ratio.

Another comparison of the scouring process between the present supercritical configuration and the more widely-studied subcritical configurations is presented here. The increase of the maximum scour depth with time, plotted in Fig. 6, is compared with the semi-empirical formula proposed by Oliveto and Hager (2002) and validated against data for subcritical conditions from a range of other studies. Using the parameters proposed by the authors for an abutment and a uniform sediment of high density and taking as subcritical approach conditions the conjugate water depth $\left(h_{0}=2.3 \mathrm{~cm}\right)$ and velocity $\left(V_{0}=0.25 \mathrm{~m} / \mathrm{s}\right)$ downstream from the hydraulic jump along the symmetry plane of the inflow, this prediction results in the continuous line shown in Fig. 6. The agreement between the predicted and measured maximum scour depth evolution with time is reasonable, at least after a few minutes, which suggests that the processes driving the scour might be similar in the two flow regimes. It would also have been interesting to compare the maximum deposition elevation plotted in Fig. 6 with the empirical formula from Oliveto and Hager (2014) fitted on subcritical configuration data from the literature. This formula involves both a water depth, which could be read directly on Fig.3, and a typical velocity, controlling the deposition, which was not measured, so it is unfortunately not possible to present this comparison. To conclude, some aspects of the scouring processes occurring in supercritical and subcritical inflow differ markedly, whilst others appear to be rather similar. 


\section{Correspondence with field conditions}

The experiments were conducting using rather heavy sediments (blasting shot) to ensure a Shields parameter slightly below the critical one; nevertheless, this does not prevent from respecting similarities with field flows. In order to determine the field conditions which are dynamically similar to those of the experiments, we consider: i) a Froude similarity with $F r^{\prime}=2.07$, ii) an arbitrary laboratory/field scale of $1 / 10$, iii) a field sediment density $\rho^{\prime}{ }_{s}=2650$ $\mathrm{kg} / \mathrm{m}^{3}$, iv) the Manning equation for the uniform incoming flow (with a Manning coefficient $n^{\prime}=d^{\prime 1 / 6} / 21.1$ as proposed by A. Strickler in 1923), v) and a critical Shields parameter $\theta_{\mathrm{cr}}$, corresponding to the incipient motion as in the experiment, with the prime sign referring to the field scale. The resulting field variables are then: for the inflow, a velocity $U^{\prime}=2 \mathrm{~m} / \mathrm{s}$, a slope $i^{\prime}=8.4 \%$ and a water depth $h^{\prime}=9.35 \mathrm{~cm}$ (leading to a Reynolds number $\mathrm{Re}^{\prime}=7.4 \times 105$; for the sediment: a mean diameter $d^{\prime}=6.5 \mathrm{~cm}$ and a Shields parameter $\theta^{\prime}=0.055$; and for the obstacle: $R^{\prime} L^{\prime}=98 \mathrm{~cm} \times 65 \mathrm{~cm}$. Finally, the time equivalent to the present $120 \mathrm{~min}$ of experiments equals 6.3 hours with a maximum scour depth of about $16 \mathrm{~cm}$ and a maximum deposition elevation of $9 \mathrm{~cm}$. In such a full scale flow the ratio of depth to sediment diameter would be very low $\left(h^{\prime} / d^{\prime}=1.4\right)$, but might be characteristic of a very shallow flow over a gravel bed interacting with a large obstacle $\left(R^{\prime} / h^{\prime}=10.5\right)$, and would be most likely to occur in torrential flow on a steep slope. So the field conditions could correspond, for example, to bridge pier in a torrential stream or river. The width of such watercourses varies quite widely, from, for example, the $520 \mathrm{~m}$ wide Rivière des Galets at Saint Etienne de la Reunion to the $10 \mathrm{~m}$ wide (50m during a flood) Mapocho river in Santiago (Chile). Now, correspondence with field conditions remains limited due mainly to the shallow depth $\left(h^{\prime} \sim 10 \mathrm{~cm}\right)$ of the incoming supercritical flow. This limited depth was imposed by the need to produce a flow in the supercritical regime but without transporting sediments from upstream, in order to focus on the role of the hydraulic jump. This approach slightly differs from the works by Jain and Fischer (1980) or Chiew (1984) who also considered an inflow in supercritical regime but under live-bed conditions. The clear-water conditions considered herein could be representative of different flow configurations in the field such as scour in a river with large sediments for which the Shields stress far from the obstacle would not exceed its critical value. Future work should be devoted to investigating scouring in more realistic supercritical flow conditions, such as live-bed conditions.

\section{Conclusions}

The aim of this research was to investigate the scouring process occurring in a steady uniform flow in the supercritical regime interacting with an emergent, impervious obstacle of rectangular shape, in a clear-water configuration. For the sake of simplicity, the selected flow configuration was at incipient motion, that is with the Shields parameter slightly below critical 
before inserting the obstacle, which required the use of heavy particles, made of steel. A specific measuring device was set up in order to measure the 2D bed topography and water surface elevation at different times through the experiment. The main results of the paper are that: - initially, the flow pattern above a flat rough bed, is similar to that observed in previous studies of flow over a smooth fixed bed with a bow-wave like detached hydraulic jump upstream from the obstacle and a very shallow -almost dry - flow region downstream.

- as time progresses, the scour begins on the sides of the obstacle and grows upstream and downstream, whilst the depth of the scour hole increases. This pattern is similar to that reported for subcritical flow configurations. Downstream of the obstacle, the deposition pattern consists of two elevated regions separated along the centerline by a much less elevated 'streamwise channel'.

- the sediment transport and topographical changes affect the hydraulic jump which, as time passes, moves towards downstream with a decreasing crest water level. Two reasons to explain the hydraulic jump displacement towards the obstacle are proposed. Downstream from the obstacle, the flow pattern is strongly affected by the deposited sediments and after one to two hours, two additional bow-waves parallel to the hydraulic jump are observed, with a constant streamwise distance of $100 \mathrm{~mm}$ between each, and with a crest elevation that decreases with downstream distance.

- finally, the hydraulic jump seems not to affect the sediment erosion. This is quite an unexpected result as, favoring the boundary layer separation, the jump was expected to participate in the scouring process, either through its action on the horseshoe vortex (see Riviere et al., 2012), or through direct turbulent structures impacting the bed (as for Bellal et al., 2003). This might be because of the rather low inlet Froude number ( $\mathrm{Fr}=2.07)$, resulting in an 'oscillating jump' (Chow, 1959); at higher Froude numbers, the impact of the jump on the scour might become stronger. Conversely, the present data reveal that it is the scouring process that seems to determine the behavior of the hydraulic jump (location, shape and crest elevation).

\section{Acknowledgments}

The authors would like to thank prof. Oscar Link from the University of Concepcion (Chile) for his advice during the writing of the manuscript.

\section{Appendix}

The measurement method is first detailed for the free-surface elevation measurement and then adaptations performed for the bed topography measurement are indicated. A laser (Z-LASER Z30M18S-F-640-11X11P) is equipped with special lenses that split the beam into 11x11 narrow beams tilted up to $28^{\circ}$ relatively to the incident laser axis. The laser is placed perpendicular to 
the flume, above the flow, and projects the grid of $N=11 \times 11$ regularly spaced points on a selected zone. A camera is positioned to one side of the flume, with its axis inclined by about $45^{\circ}$ relative to the horizontal (see Fig.11a). The image taken by the camera in the measurement zone with no water $(z=0)$ is called the reference image (labeled ' 0 ') and contains $N$ points $M_{j}{ }^{0}(j=1 \ldots N)$ with coordinates in the camera image frame (labeled ' $i$ ') noted $\left(x_{i}(j, 0), y_{i}(j, 0)\right)$. Once water is flowing in the flume, each laser beam $j$ will intersect the free surface at a point $P_{j}^{h}$ at coordinates $(x, y, h)$ with local elevation $z_{w}(x, y)$ in the frame of the flume. Each luminous point $P_{j}^{h}$ is viewed on the camera image as a spot, a few pixels wide, with center $M_{j}^{h}$ and coordinates $\left(x_{i}(j, h), y_{i}(j, h)\right)$ in the image frame (sensor plane on Fig 11b).

The principle of the method relies on two basic optical effects. Firstly, on the camera image any image point $M_{j}^{h}$ exhibits a deviation $\left[x_{i}(j, h)-x_{i}(j, 0)\right]$ (and $\left[y_{i}(j, h)-y_{i}(j, 0)\right]$ ) which is proportional to the local water elevation $z_{w}$ (x,y) (Fig. 11b) with a coefficient denoted $\alpha_{\mathrm{j}}$ that depends on the laser beam $j$. Using this property, the $N$ coefficients $\alpha_{j}$ are inferred from a calibration procedure performed by projecting the laser onto calibration plates of known thickness. Secondly, due to the camera angle and the large depth of field, length distortion has to be considered: for a regular laser grid, the further two points are from the camera focal point, the closer to each other they appear on the photo. This effect depends on the surface elevation. A second calibration procedure is thus needed (without the laser) to establish the relationship between the $(x, y)$ coordinates of the real intersection point of the laser beam with the free-surface and the image in-plane coordinates. We use a target drawn on several calibration plates, consisting of regularly spaced points of known actual coordinates $\left(x, y, z_{w}\right)$. Image processing and linear interpolation are applied to determine a projection mapping $\left(x_{i}\left(z_{w}\right), y_{i}\left(z_{w}\right), z_{w}\right) \rightarrow\left(x, y, z_{w}\right)$. After calibration (determination of the $\alpha_{j}$ and of the projection mapping), for any image of the grid projected on the free surface, the measurement procedure is as follows. First, the image coordinates $\left(x_{i}, y_{i}\right)$ of the centers of the $N$ spots $M_{j}^{h}$ are determined by image processing. Second, the water depth at each $\mathrm{N}$ point is calculated using $h\left(x_{i}(j), y_{i}(j)\right)=\left[x_{i}(j)-x_{i}(j, 0)\right] / \alpha_{\text {j. }}$. Third, knowing $h$ for each $M_{j}^{h}$, the real in-plane coordinates $(x, y)$ of the water surface/laser intersections $P_{j}^{h}$ are inferred from the projection mapping $\left(x_{i}(h), y_{i}(h), h\right) \rightarrow(x, y, h)$. Fourth, the water depth map (as presented in the results section) is deduced from linear interpolation of the actual coordinates $(x, y, h)$ of the $N$ measured points.

The principle used to obtained the sediment elevation maps is similar to that described for the free-surface except that the calibration plates are located at elevations ranging from the fixed bottom $(z=-20 \mathrm{~mm})$ to the maximum sediment deposition elevation: $z \sim 10 \mathrm{~mm})$.

519 Ahmed F. and Rajaratnam N. (1998), Flow around bridge piers, J. Hydraul. Eng., 124(3), 288-300. 
Bellal M., Spinewine B., Savary C. and Zech Y. (2003), Morphological Evolution of Steep-Sloped River Beds in the Presence of a Hydraulic Jump: Experimental Study, Proceedings of XXX IAHR Congress, Thessaloniki, Greece, August 2003, Vol. C-II, 133-140.

Chiew Y. M., (1984), Local scour at bridge piers, Rep. No. 355, Dept. of Civil Engineering, Univ. of Auckland, Auckland, New Zealand.

Chow, V. T. (1959), Open channel hydraulics, McGraw-Hill, New York.

Dargahi B. (1989), The turbulent flow field around a circular cylinder, Exp. Fluids, 8(1-2), 1-12.

Dey S. and Raikar R.V. (2007), Characteristics of Horseshoe Vortex in Developing Scour Holes at Piers, Journal of Hydraulic Engineering, Vol. 133(4), 399-413

Diab R., Link O. and Zanke U. (2010), Geometry of developing and equilibrium scour holes at bridge piers in gravel, Canadian J. of Civil Eng., 37(4), 544-552.

Escauriaza C. and Sotiropoulos F. (2011), Reynolds number effects on the coherent dynamics of the turbulent horseshoe vortex system, Flow Turbul. Combust., 86, 231- 262.

Gobert C., Link O., Manhart M. and Zanke U. (2010), Discussion of "Coherent Structures in the Flow Field around a Circular Cylinder with Scour Hole" by G. Kirkil, G. Constaninescu, and R. Ettema, J. Hydraul. Eng, 136(1), 82-84.

Graf W. H. and Yulistiyanto B. (1998), Experiments on flow around a cylinder; the velocity and vorticity fields, J. Hyd. Research., 36(4), 637-653.

Hager W.H. and Unger J. (2010), Bridge Pier Scour under Flood Waves J. Hydraul. Eng., 136(10), $842-847$

Ippen A. T. (1951), Mechanics of supercritical flow: 1st paper of high velocity flow in open channels: A symposium, Transactions ASCE 116, 268.

Jain S.C. and Fischer E.E. (1980), Scour around bridge piers at high velocities, J. of the Hydraul. Division, 106(11), 1827-1842.

Jiang Q. and Smith R. B. (2000), V-waves, bow shocks, and wakes in supercritical hydrostatic flows, J. Fluid Mech., 406, 27-53. 
Kirkil G., Contantinescu G. and Ettema R. (2008), Coherent Structures in the Flow Field around a Circular Cylinder with Scour Hole, J. Hydraul. Eng, 134(5), 572-587.

Lai J.-S., Chang W.Y. and Yen C.-L. (2009), Maximum Local Scour Depth at Bridge Piers under Unsteady Flow, J. Hydraul. Eng, 135(7), 609-614.

Link O., Gonzalez C., Maldonado M. and Escauriaza C. (2012), Coherent Structure Dynamics and Sediment Particle Motion Around a Cylindrical Pier in Developing Scour Holes, Acta Geophysica, vol. 60(6), 1689-1719

Lipeme Kouyi G., Vazquez J. and Poulet J.B. (2003), 3D free surface measurement and numerical modelling of flows in storm overflows, Flow Measurement and Instrumentation, 14(3), 7987.

Melville B.W., and Chiew Y.M.(1999), Time scale for local scour at bridge piers, Journal of Hydraulics Engineering, 125(1), 59-65.

Mignot E. and Riviere N. (2010), Bow-wave like hydraulic jump and horseshoe vortex around an obstacle in a supercritical open channel flow, Physics of Fluids, 22, 117105.

Mignot E., Riviere N. and Chakraverty K. (2011),Detached hydraulic jump upstream a fixed obstacle in supercritical flow. 34th IAHR Congress, 26 June to 1st July 2011, Brisbane, Australia.

Moeckel W. E. (1949), Approximate method for predicting form and location of detached shock waves ahead of planar or axially symmetric bodies, Technical Note 1921, National Advisory Committee for Aeronautics, p. 32.

Muzzammil M. and Gangadhariah T. (2003), The mean characteristics of horseshoe vortex at a cylindrical pier, Journal of Hydraulic Research, 41(3), 285-297

Oliveto G. and Hager W.H. (2002), Temporal evolution of clear-water pier and abutment scour, Journal of Hydraul. Eng., 128(9), 811-820.

Oliveto G. and Hager W.H. (2014), Morphological Evolution of Dune-Like Bed Forms Generated by Bridge Scour, J. Hydraul. Eng., 140(5), CID:06014009. 
572 Riviere N., Laily A.-G., Mignot E. and Doppler D. (2012), Supercritical flow around and beneath a 573 fixed obstacle, Proceeding of the 2nd IAHR Europe Conference, 27.-29. June 2012, Munchen, $574 \quad$ Germany.

575 Roulund A., Sumer M., Fredsoe J., and Michelsen J. (2005), Numerical and experimental 576 investigation of flow and scour around a circular pile, J. Fluid Mech., 534, 351-401.

577 Sadeque M. A., Rajaratnam N., and Loewen M. R. (2008), Flow around cylinders in open channels, $578 \quad$ J. Eng. Mech., 134(1), 60-71.

579 Sahin B., Ozturk N. A., and Akilli H. (2007), Horseshoe vortex system in the vicinity of the vertical 580 cylinder mounted on a flat plate, J. Fluid Mech., 18(2), 57-68.

581 Shapiro A. H. (1953), The Dynamics and Thermodynamics of Compressible Fluid Flow, Ronald, New York, 881-888.

Sheppard M. and Miller W. (2006), Live-Bed Local Pier Scour Experiments, J. Hydraul. Eng., 132(7), 635-642.

Unger J. and Hager W.H. (2007), Down-flow and horseshoe vortex characteristics of sediment embedded bridge piers, Exp Fluids 42(1), 1-19. 